\title{
Detection of DNA damage by space radiation in human fibroblasts flown on the International Space Station
}

Tao Lu, Ye Zhang, Michael Wong, Alan Feiveson, Ramona Gaza, Nicholas Stoffle, Huichen Wang, Bobby Wilson, Larry Rohde, Louis Stodieck, Fathi Karouia, and Honglu Wu

\begin{abstract}
Although charged particles in space have been detected with radiation detectors on board spacecraft since the discovery of the Van Allen Belts, reports on the effects of direct exposure to space radiation in biological systems have been limited. Measurement of biological effects of space radiation is challenging due to the low dose and low dose rate nature of the radiation environment, and due to the difficulty in distinguishing the radiation effects from microgravity and other space environmental factors. In astronauts, only a few changes, such as increased chromosome aberrations in their lymphocytes and early onset of cataracts, are attributed primarily to their exposure to space radiation. In this study, cultured human fibroblasts were flown on the International Space Station (ISS). Cells were kept at $37^{\circ} \mathrm{C}$ in space for 14 days before being fixed for analysis of DNA damages with the $\gamma-\mathrm{H} 2 \mathrm{AX}$ assay. The 3-dimensional $\gamma$ H2AX foci were captured with a laser confocal microscope. Quantitative analysis revealed several foci that were larger and displayed a track pattern only in the Day 14 flight samples. To confirm that the foci data from the flight study was actually induced from space radiation exposure, cultured human fibroblasts were exposed to low dose rate gamma rays at $37{ }^{\circ} \mathrm{C}$. Cells exposed to chronic gamma rays showed similar foci size distribution in comparison to the nonexposed controls. The cells were also exposed to low- and high-LET protons, and high-LET Fe ions on the ground. Our results suggest that in G1 human fibroblasts under the normal culture condition, only a small fraction of large size foci can be attributed to high-LET radiation in space.
\end{abstract}

